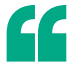

aninflammatory

pathway in ageing that is triggered by gut dysbiosis, leading to ... activated monocytes in the omentum and $4 \mathrm{BL}$ cell conversion

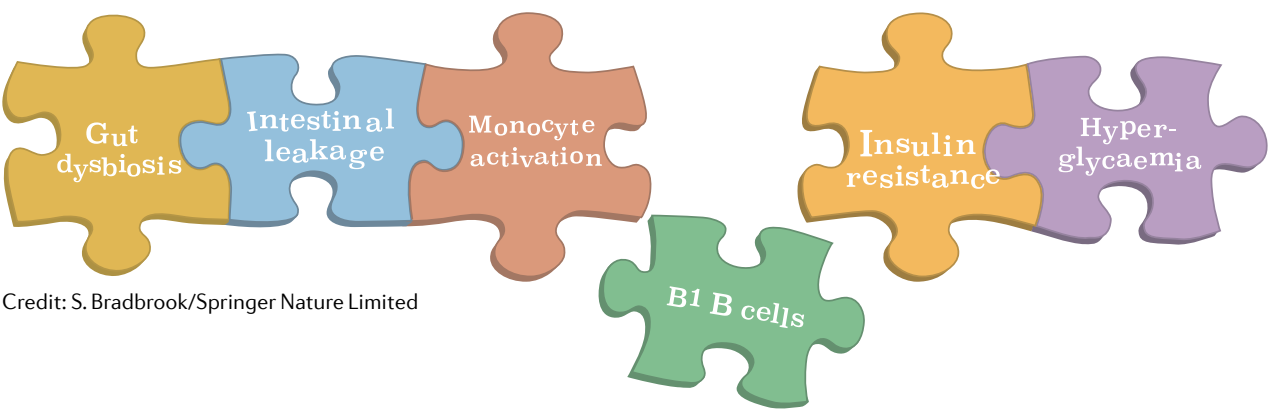

Changes to the gut microbiota during ageing can be associated with chronic inflammation and, therefore, possibly with pathologies such as hyperglycaemia and insulin resistance (IR). Bodogai et al. now show that innate B1a B cells might be the missing link in this pathway.

The authors previously reported that aged humans, primates and mice accumulate innate B1a B cells that express 4-1BBL (also known as TNFSF9) and TNF - termed 4BL cells - in the peritoneal cavity. In the current study, they showed that aged mice also accumulate CCR2 ${ }^{+}$ monocytes in the peritoneal cavity that express 4-1BB (also known as TNFRSF9), CD40L and IFN $\gamma$, the three factors involved in 4BL cell induction. Monocytes colocalized with $4 \mathrm{BL}$ cells in the omentum of aged mice, and co-culture of B1a $B$ cells from young mice with omentum from aged mice led to the upregulation of 4-1BBL expression by the B cells. The $4 \mathrm{BL}$ cell conversion of B1a B cells from young mice adoptively transferred to aged mice was prevented by treatment with an antibody that depletes CCR2 ${ }^{+}$ monocytes. The authors conclude that $4 \mathrm{BL}$ cells are induced by monocytes in the aged omentum.

Next, they linked the induction of $4 \mathrm{BL}$ cells to the gut microbiota. The accumulation of monocytes and 4BL cells in the omentum of aged mice was abolished by treatment with the broad-spectrum antibiotic enrofloxacin, and antibiotic-treated aged mice could not mediate the 4BL cell conversion of donor B1 B cells from young mice. The gut microbiota of aged mice was enriched for pro-inflammatory bacterial phyla and had reduced abundance of potentially beneficial commensals such as Akkermansia muciniphila and butyrate-producing Firmicutes. When adult germ-free mice were colonized with faecal suspensions from young and aged mice, only the latter had increased numbers of monocytes and 4BL cells in the omentum. Thus, the aged gut microbiota triggers monocyte-mediated 4BL cell conversion.

A. muciniphila supports integrity of the intestinal barrier; in keeping with this, aged mice (with decreased abundance of $A$. muciniphila) had a thinner colonic mucus layer, increased intestinal permeability and increased serum levels of endotoxin. Treatment with A. muciniphila or enrofloxacin (which increased the abundance of A. muciniphila, presumably owing to antibiotic resistance) reversed the intestinal barrier dysfunction in aged mice and prevented the induction of $4 \mathrm{BL}$ cells.

The inflammatory response to translocation of bacterial products across the dysfunctional intestinal barrier of aged mice might be enhanced by the decreased levels of immunoregulatory butyrate found in these mice. For mouse monocytes stimulated with lipopolysaccharide (LPS) in vitro, the addition of butyrate

MICROBIOTA

\title{
B1 B cells link gut dysbiosis and insulin resistance
}

prevented the upregulation of 4-1BBL by B1 B cells. Treatment of aged mice with butyrate prevented $4 \mathrm{BL}$ cell conversion of host B1 B cells and donor B1 B cells from young mice.

Finally, the authors linked increased numbers of 4BL cells with IR in aged mice. Enrofloxacin abolished the increased IR seen in healthy aged mice, as did treatment with butyrate, or the depletion of $4 \mathrm{BL}$ cells or CCR2+ monocytes. B cell-deficient mice that were metabolically sensitized by a high-fat diet were replenished with B1a B cells from young mice or $4 \mathrm{BL}$ cells from aged mice; IR was increased only in the latter group of mice. Treatment with A. muciniphila abolished the increased IR in aged mice; this beneficial effect was reversed by the introduction of $4 \mathrm{BL}$ cells, but not 4-1BBL-knockout 4BL cells.

The study also suggests that this pathway can occur in aged humans and non-human primates, as butyrate blocked the ability of LPS to convert human monocytes into 4BL cell inducers. Enrofloxacin also abrogated monocyte-mediated 4BL cell conversion in aged macaques and reversed IR.

Together, the results suggest an inflammatory pathway in ageing that is triggered by gut dysbiosis, leading to intestinal leakage, accumulation of activated monocytes in the omentum and $4 \mathrm{BL}$ cell conversion. 4BL cells then induce IR in a manner dependent on 4-1BBL, a factor that has been implicated in obesity-induced adipose tissue inflammation. Importantly, the authors show that this process is reversible in aged mice and macaques, which opens up new therapeutic options.

Kirsty Minton

ORIGINAL ARTICLE Bodogai, M. et al. Commensal bacteria contribute to insulin resistance in aging by activating innate B1a cells. Sci. Transl Med. 10, eaat4271 (2018) 